\section{Pumping plaques away}

\section{By Lev Osherovich, Senior Writer}

A study by Medtronic Inc. makes a case for using pumps implanted in the brain for the delivery of antibodies against $\beta$-amyloid, the plaqueforming protein responsible for the pathology of Alzheimer's disease. ${ }^{1}$ Intracerebroventricular infusion of the antibodies could avoid some of the vascular side effects that have marred trials of systemic $\beta$-amyloid immunotherapies.

If the method can be used to deliver antibodies into the brains of large animals, it could move quickly into the clinic, according to Lisa Shafer, lead author on the paper in the Proceedings of the National Academy of Sciences and a principal scientists at Medtronic.

As reported in PNAS, the Medtronic team used a subcutaneous osmotic mini-pump to administer $\beta$-amyloid $(\mathrm{A} \beta)$ antibodies directly

"This suggests that the
removal of A $\beta$ from the
arterial walls should be
attempted in a slow,
gradual manner."
- Roxana Carare,
University of Southampton

vaccination, conventional $\mathrm{mAb}$ therapy can also elicit cerebral amyloid angiopathy. ${ }^{4}$

The Medtronic study suggests a work-around that could render the administration of $A \beta-m A b s$ safer and more effective by lowering the effective doses.

\section{Into the brain}

Using a mouse model of $\mathrm{AD}$ that produces excess amounts of $\mathrm{A} \beta$, the Medtronic team gave a preclinical murine $\mathrm{mAb}$ against $\mathrm{A} \beta$ systemically or by $i c v$ perfusion from implanted mini-pumps. Because icv delivery bypasses the need for high systemic mAb concentrations in order to deliver therapeutically relevant concentrations across the blood-brain barrier (BBB), the icv-treated mice received 10-50-fold less total antibody than systemically treated animals.

After five weeks, and despite the dosage difference, icv-treated and systemically treated mice had similar decreases in parenchymal $A \beta$ levels and less cognitive decline compared with what was seen in mock-treated controls.

The icv-treated mice also showed less cerebral amyloid angiopathy than mock-treated controls, and systemically treated mice had higher proportions of their brains affected by cerebral amyloid angiopathy than did controls.

"Our findings relative to systemic administration replicate the plaque-clearing effect and the angiopathy of these earlier studies, but we into the intracerebroventricular (icv) space, thereby avoiding cerebral amyloid angiopathy, a dangerous side effect of systemic $A \beta$ immunotherapy.

Most $A \beta$ plaques are found in the brain parenchyma around the neurons. In cerebral amyloid angiopathy, $\mathrm{A} \beta$ plaques accumulate in the microvasculature surrounding neurons, leading to microhemorrhages and strokes. ${ }^{2}$

According to Donna Wilcock, assistant professor of neurology at Duke University School of Medicine, both parenchymal and vascular plaque deposition can potentially contribute to neuroinflammation and cognitive dysfunction in $\mathrm{AD}$.

Cerebral amyloid angiopathy is already a significant component of $\mathrm{AD}$ pathology, but the condition appears to increase as a result of $\mathrm{A} \beta$ immunotherapy, a problem that may have contributed to the failure of Wyeth and Elan Corp. plc's AN-1792 A $\beta$ vaccine in 2002. Indeed, postmortem analysis of patients from the AN-1792 trial suggested that treatment actually increased cerebral amyloid angiopathy compared with that seen in untreated controls. ${ }^{3}$

Elan and Wyeth are now testing a second-generation vaccine, ACC-001, which is in Phase II trials, and are also pursuing AD with bapineuzumab, a mAb against $\mathrm{A} \beta$. Although the antibody missed the endpoints of a Phase II trial last fall, the companies are now running a Phase III study using a different patient stratification scheme.

The switch in treatment modality may not be enough to avoid the unwanted vascular effect, however. Preclinical studies suggest that, like demonstrate that $i c v$ is also effective in clearing plaques without eliciting angiopathy," said Shafer.

"I find these data very encouraging," said Wilcock. "Microhemorrhage does appear to be a consequence of systemic immunotherapy. This is why the current study is so important-it shows that there is a route of administration of antibodies that will avoid this adverse event."

David Cribbs, associate professor of neurology at the University of California, Irvine, suggested that continuous, low-dose treatment with antibody was the key to Medtronic's results with the pump. Not only did the icv mice receive less total antibody than systemically treated mice, but also they received the antibody continuously over five weeks rather than the five weekly, high-dose injections in the systemically treated group.

"It's not that there's something magical about icv, but rather they are able to use a lower dose to get plaque clearance," said Cribbs.

Roxana Carare, lecturer in clinical neurosciences at the University of Southampton, told SciBX that slow, low-dose delivery may facilitate the drainage of amyloid-antibody complexes out of the brain. A recent study by Carare suggested that, in systemic immunotherapy, bulky complexes of antibodies bound to $A \beta$ get stuck in the surrounding vascular tissue. $^{5}$

Clearance of plaques by icv immunotherapeutics "may be slow enough to dislodge the $A \beta$ that is entrapped in the perivascular drainage pathways along the basement membranes," said Carare. "This suggests that the removal of $A \beta$ from the arterial walls should be attempted in a slow, gradual manner." 


\section{Scaling up}

The antibody used in the PNAS study recognizes the same portion of the $A \beta$ protein as bapineuzumab but is not optimized for human use. Thus, if Medtronic pursues an IND for icv immunotherapy in AD, the company probably will need to partner with an antibody company.

In addition to bapineuzumab, the $\mathrm{A} \beta$ antibody space includes at least five other compounds in clinical development. RN1219 from Pfizer Inc.'s Rinat Neuroscience Corp. subsidiary, R1450 from MorphoSys AG and partner Roche, Genentech Inc.'s MABT5102 and GlaxoSmithKline plc's GSK933776A are all in Phase I trials for AD. PF-04360365, from Pfizer, started a Phase II AD trial last fall. Pfizer is acquiring Wyeth, and Roche is acquiring Genentech.

Shafer did not disclose which antibody candidates Medtronic was considering.

Another question is whether the technique will scale-up in larger brains. It is not clear whether antibodies administered to the ventricles can penetrate deep enough into the parenchyma to clear out plaques. Thus, before filing an IND, Shafer said further preclinical studies of brain penetration by $i c v$-delivered antibodies may need to be performed.

"A key point for the IND-enabling studies will be to gain a better understanding of the distribution" of the antibody throughout the brain, said Shafer. "We need to do this with larger animals."

NeuroNova AB is using Medtronic's pump and catheter devices in clinical trials of its treatments for amyotrophic lateral sclerosis (ALS) and Parkinson's disease (PD). CSO Anders Haegerstrand was "quite optimistic about the overall approach" in AD.

Haegerstrand cautioned that treating $\mathrm{AD}$ with an implanted device might pose some specific challenges because of concerns about compliance by frail or demented patients.

"An implantation procedure of catheter and pump, involving neurosurgery, could be considered too complicated and demanding to many patients and relatives, even though the benefits can be great," he said. "Implantation of medical devices into patients that are demented poses some potential difficulties if they are bothered by the device, which can be felt through the skin. They might tamper with the pump and catheter from the outside. Starting such a therapy relatively soon after diagnosis might be important to circumvent these issues."
NeuroNova's sNN0029, an icv-delivered formulation of VEGF, is in a Phase I trial for ALS. This month, the company began a Phase I trial of sNN0031, a platelet derived growth factor B (PDGFB) dimer, in PD. Both trials use the Medtronic technology.

Compliance caveats aside, Haegerstrand thinks icv delivery of NeuroNova's VEGF formulation potentially could be helpful for AD, especially in combination with immunotherapeutics. He said NeuroNova is awaiting safety data from the ALS and PD trials before considering whether to proceed into the $\mathrm{AD}$ space.

Medtronic has also partnered with Alnylam Pharmaceuticals Inc. for $i c v$ delivery of small interfering RNA therapeutics to treat Huntington's disease (HD).

The method and devices are covered by pending patents from Medtronic. The company already has icv pumps on the market for pain and spasticity indications.

Osherovich, L. SciBX 2(12); doi:10.1038/scibx.2009.474

Published online March 26, 2009

\section{REFERENCES}

1. Thakker, D.R. et al. Proc. Natl. Acad. Sci. USA; published online Feb. 25, 2009; doi:10.1073/pnas.0813404106

Contact: Lisa L. Shafer, Medtronic Inc., Minneapolis, Minn. e-mail: lisa.I.shafer@medtronic.com

2. Wilcock, D.M. \& Colton, C.A. CNS Neurol. Disord. Drug Targets 8, 50-64 (2009)

3. Boche, D. et al. Brain 131, 3299-3310 (2008)

4. Wilcock, D.M. et al. J. Neuroinflammation 1, 24 (2004)

5. Carare, R.O. et al. Neuropathol. Appl. Neurobiol. 34, 131-144 (2008)

COMPANIES AND INSTITUTIONS MENTIONED

Alnylam Pharmaceuticals Inc. (NASDAQ:ALNY), Cambridge, Mass. Duke University School of Medicine, Durham, N.C.

Elan Corp. plc (NYSE:ELN), Dublin, Ireland

Genentech Inc. (NYSE:DNA), South San Francisco, Calif. GlaxoSmithKline plc (LSE:GSK; NYSE:GSK), London, U.K.

Medtronic Inc. (NYSE:MDT), Minneapolis, Minn.

MorphoSys AG (Xetra:MOR), Martinsried, Germany

NeuroNova AB, Stockholm, Sweden

Pfizer Inc. (NYSE:PFE), New York, N.Y.

Roche (SIX:ROG), Basel, Switzerland

University of California, Irvine, Calif.

University of Southampton, Southampton, U.K.

Wyeth (NYSE:WYE), Madison, N.J. 